\title{
Autonomieverlust der Medizin? Zum Strukturwandel moderner Großkrankenhäuser in Deutschland, Österreich und der Schweiz
}

\section{Zusammenfassung}

Der Beitrag untersucht im Ländervergleich zwischen Deutschland, Österreich und der Schweiz, inwiefern sich die Strukturen der großen Krankenhäuser in Richtung einer Ökonomisierung, verstanden als zunehmende Geltung von Wirtschaftlichkeitskriterien, verändern. Der mögliche Autonomieverlust der Medizin durch betriebswirtschaftliches Management und betriebswirtschaftliche Effizienzprinzipien infolge dieser institutionellen Weichenstellungen kann Folgen für die Konstellation in den Arbeitsbeziehungen im Krankenhaus zeitigen. Auf Basis von Struktur-, Lebensverlaufs- und Inhaltsanalysen lässt sich erkennen, dass die Veränderung der institutionellen Verfasstheit der Großkrankenhäuser und der Leitungs- und Qualifikationsstrukturen je nach länderspezifischem Kontext unterschiedlich stark dafür sorgen, dass der konservative Pfad durchbrochen wird und die institutionellen Weichenstellungen in Richtung zunehmender Ökonomisierung zeigen: in der Schweiz am weitreichendsten, in Österreich am geringsten.

Schlagwörter: Öffentliche Krankenhäuser, Ökonomisierung, Verbetrieblichung, De-Professionalisierung

\section{Loss of autonomy of medicine? Managerialism in modern large hospitals in Germany, Austria and Switzerland}

\begin{abstract}
A comparative study of Germany, Austria and Switzerland, the article discusses the question of how far current changes in public hospitals have induced a shift towards commodification and managerialism. As a consequence of the increasing importance of management, business and efficiency principles, resulting in a decline in medicine's autonomy, labour relations in hospitals are likely to be effected. Based on structural analysis, life course analysis and content analysis the findings indicate how changes in the management and qualification structure of large hospitals have resulted in them
\end{abstract}

\footnotetext{
* Dr. Stefan Bär \& Prof. Dr. Markus Pohlmann, beide Universität Heidelberg, Max-Weber-Institut für Soziologie, Bergheimer Straße 58, D-69115 Heidelberg.

E-Mail: stefan.baer@soziologie.uni-heidelberg.de, markus.pohlmann@soziologie.uni-heidelberg.de.

** Artikel eingegangen: 03.08.2016, revidierte Fassung akzeptiert nach doppelt-blindem Begutachtungsverfahren: 30.06 .2017
} 
abandoning their conservative path. To varying degrees commodification is modifying the institutional structures of large hospitals in all three countries. Whilst Switzerland can be shown to be setting the commodification pace, such a process has had the least impact in Austria.

Keywords: Public hospitals, commodification, managerializiation, de-professionalization (JEL: I11, I18, J53, 057)

\section{Einleitung}

Die Krankenhauslandschaft in Deutschland, Österreich und der Schweiz hat sich in den letzten beiden Dekaden drastisch verändert. Die ausgedehnte Privatisierung öffentlich getragener Krankenhäuser ist lediglich einer von vielen Indikatoren dafür (vgl. z.B. Statistisches Bundesamt, 2016). Medizinischer Fortschritt, Hightech-Medizin und die Behandlung immer älterer, multimorbider Patientinnen und Patienten führen zu ständig steigenden Kosten in der stationären Krankenversorgung. Regelmäßig stehen sie bzw. deren Eindämmung daher im Mittelpunkt der Gesundheitspolitik (Simon, 2016, S. 30ff.). Dadurch steigt der Druck in Richtung effizientes Wirtschaften für die einzelnen Krankenhäuser. Die neuen Abrechnungs- und Fallpauschalensysteme ${ }^{1}$ sowie das New Public Management tragen das ihre dazu bei. Die Spannungslinien im Feld der öffentlich getragenen Großkrankenhäuser laufen jedoch nicht einfach zwischen Gemeinwohlverpflichtung und Profitmaximierung, sondern zwischen medizinischer Effektivität, bürokratischer Organisation und betriebswirtschaftlicher Effizienz und sind damit grundlegender: zwischen der fachlich-professionellen Autonomie der Ärzteschaft einerseits und ihrer heteronomen Stellung als Personal im Krankenhausbetrieb andererseits.

Dies beinhaltet auf der Ebene der Arbeitsbeziehungen typische Probleme, wie jene zwischen der Fach-, Reputations- und Karriereorientierung der Professionsangehörigen und ihrer Stellung, ihrer administrativen und betriebswirtschaftlichen Funktion im Krankenhaus. Und auch die professionelle Verpflichtung, das eigene Leben, nicht nur die Arbeit, in den Dienst von Krankenbehandlung und Patientenwohl zu stellen, schafft eine andere, als in Unternehmen übliche Konstellation in den Arbeitsbeziehungen im Krankenhaus. ${ }^{2}$ Diese professionell-ethisch untermauerte Entgrenzung von Arbeit verschafft der Organisation einen erweiterten Zugriff auf die Ressource der Arbeitskraft, so dass sich die Arbeitskonflikte klassischer Weise an der Überdehnung dieses Zugriffs sowie an der Kompensation für die In-Dienst-Stellung des gesamten Lebens entzünden. Da die öffentliche Hand nur in Ausnahmefällen und lediglich für wenige Spitzenpositionen Kompensationen wie in der Privatwirtschaft zur Verfügung stellt, sind Autonomie, Zeit und Entlohnung ständig wiederkehrende Kampffelder in den Arbeitsbeziehungen. Unser Artikel konzentriert sich auf lediglich eines davon: das Kampffeld der Autonomie der Profession, und fragt, durch welche

1 Mit der Einführung von Fallpauschalensystemen wie dem der Diagnosis Related Groups (DRG) wird zum einen die Finanzierung der laufenden Krankenhauskosten über tagesgleiche Pflegesätze durch eine Art Preissystem ersetzt und zum anderen die ökonomische Bewertung von Behandlungen gekoppelt an die bei einer Diagnose im Durchschnitt entstehenden, und nicht an die tatsächlich anfallenden Kosten (vgl. hierzu Simon 2013a, S. 416ff.).

2 Das Gelöbnis der Ärzteschaft besagt dazu: „Bei meiner Aufnahme in den ärztlichen Berufsstand gelobe ich, mein Leben in den Dienst der Menschlichkeit zu stellen“ (Schott, 2006). 
strukturellen Veränderungen im Krankenhaus der Autonomieanspruch der Profession tangiert wird. Wir begeben uns damit auf die Suche nach den institutionellen Weichenstellungen für einen Wandel in den Arbeitsbeziehungen des öffentlichen Sektors an einer ganz spezifischen Stelle: in Großkrankenhäusern. Erst mit der Aufklärung dieses Hintergrunds lassen sich Veränderungen in den kollektiven Deutungs- und Handlungsorientierungen der Ärzteschaft als mögliche Reaktionen auf einseitig veränderte Arbeitsbeziehungen verstehen. Der Streitpunkt ist beim Wandel des Krankenhauses immer wieder, inwiefern Kennzahlensysteme, Dokumentationspflichten und Abrechnungsmodi das Mandat zur autonomen Problembearbeitung der Profession aushöhlen und zu einer schlechteren Krankenbehandlung führen (Schmitz \& Berchtold, 2016, S. 95ff.). Der Artikel setzt unabhängig von deren Bewertung zunächst konkret bei der Frage an, inwiefern sich die Strukturen der Großkrankenhäuser in Richtung einer Verbetrieblichung und Managerialisierung verändert haben. Zugespitzt gefragt: Haben wir es mit einem Autonomieverlust der Medizin durch Management und betriebswirtschaftliche Effizienzprinzipien zu tun oder bleibt der medizinische Kern der professionellen Organisation intakt?

Im ersten Schritt skizzieren wir zunächst unseren theoretischen Ausgangspunkt sowie den Stand der Forschung und stellen das Forschungsdesign vor (2), um uns dann auf zwei Ebenen mit der Fragestellung zu beschäftigen: Vor dem Hintergrund des Wandels der institutionellen Ordnungen untersuchen wir einige der für die Veränderung der Arbeitsbeziehungen wichtigen strukturellen Konstellationen von Großkrankenhäusern (3). Wir wählen dabei den Ausgangspunkt in Deutschland und vergleichen (4) die Befunde mit der Situation in Österreich und der Schweiz, um den jeweiligen Einfluss des institutionellen Kontextes abschätzen zu können.

\section{Theorie, Stand der Forschung und Methode}

Denkt man das Krankenhaus von der medizinischen Profession - als einer exklusiven Berufsgruppe (Abbott, 1988) - aus, dann stellt sich die Frage, ob sich mit dem Zurückdrängen der Professionsansprüche durch das Management die Arbeitsziehungen zu klassischen industriellen Beziehungen auf der Betriebsebene (Trinczek, 2010) wandeln und sich die Großkrankenhäuser zu post-professionellen Organisationen (Klatetzki \& Nokielski, 2010) entwickeln. Die zu prüfende Annahme dabei ist, dass die Managerialisierung der Organisation Krankenhaus zu einer De-Professionalisierung der Medizin - verstanden als Begrenzung der medizinischen Autonomie durch ökonomische Vorgaben sowie eine zunehmende Dominanz der kaufmännischen Krankenhausleitung - führt.

\subsection{Theoretische Anleitung}

Diese Annahme wird von uns auf der Strukturebene geprüft. Wir rücken damit in einem institutionentheoretischen Zugang die institutionellen Weichenstellungen im Sinne von regulativen Institutionen in den Vordergrund. Wir konzentrieren uns damit auf die Formalstrukturen, Ordnungen und Vorgaben der Organisation und unterscheiden diese von den sozial geteilten Konzeptionen von der Welt (kognitive Institutionen) sowie den internalisierten Normen und Werten (normative Institutionen) in der Organisation (Scott, 2001, S. 57). 
Letztere stehen in diesem Artikel nicht im Vordergrund, werden jedoch in Diskussion und Fazit einbezogen.

Um nun die Veränderungsdynamik auf der Strukturebene genauer bestimmen zu können, setzen wir an der Theorie professioneller Organisationen an. Mit ihr gehen wir davon aus, dass neben bürokratisch-managerialen in den Kliniken auch professional-kollegiale Koordinations- und Kontrollformen große Relevanz haben. Das Spannungsfeld ist zum einen dadurch definiert, dass sich - aus ihrem professionellen Anspruch und als überlegen erachtetem Fachwissen heraus - insbesondere die medizinischen Führungskräfte den bürokratischen Interventionen des Managements widersetzen können. Potenziale solch erfolgreicher Widerständigkeit gründen, so Iseringhausen und Staender, ,in der Unverzichtbarkeit professioneller Kompetenz, der medizinischen Einzelfallorientierung und der Interaktionsabhängigkeit der Arbeitsergebnisse" (Iseringhausen \& Staender, 2012, S. 195; vgl. dazu auch Wilkesmann 2016, S. 227ff.). Zum anderen versuchen Verwaltungskräfte und kaufmännische Führungskräfte immer wieder durch organisatorische Regeln die Profession zu organisieren, sie in die Arbeitsorganisation als heteronomes Personal einzuordnen. Da eine Arbeitsorganisation im Prinzip eine der Profession entgegengesetzte Koordinationsform ist, weil sie professionelle Handlungsorientierungen nicht nur ermöglicht, sondern auch begrenzt, ist in professionellen Organisationen dieses Spannungsfeld auf Dauer gestellt. Dabei bestimmt sich im Klinikum die medizinische Professionalität u.a. durch die formale Zuweisung und tatsächliche Inanspruchnahme eines Mandats zur autonomen Problembearbeitung. Konkret übersetzt sich diese Professionalität auch in Formen der Arbeitsorganisation, welche den medizinischen Expertinnen und Experten die relative Autonomie, den Einzelfallbezug und die Orientierung an Wissensstandards und Ethik der Expertengemeinschaft ermöglichen. De-Professionalisierung bedeutet in diesem Kontext also eine stärkere Geltung von Organisationskriterien und eine fortscheitende Organisierung der Krankenbehandlung (hierzu Vogd, 2011). Dies kann sich in den Arbeitsverträgen, in der Managerialisierung der Leitung, in der zunehmenden Bedeutung von Effizienzkriterien etc. niederschlagen und ist keineswegs per se als schlecht oder gut zu beurteilen. Dasselbe gilt auch im Falle „der“ Ökonomisierung. Sie ist lediglich eine Dimension der Organisierung der medizinischen Dienstleistung. Die Frage ist hier immer, wie stark das Kriterium der Wirtschaftlichkeit im Konfliktfall gegenüber dem Kriterium der (professionell definierten) medizinischen Angemessenheit der Krankenbehandlung Geltung beanspruchen kann und umgekehrt. Doch auch auf der Strukturebene der regulativen Institutionen stellt sich die Frage, inwiefern in der institutionellen Ordnung der Krankenhäuser die Voraussetzungen geschaffen wurden, dass das Kriterium der Wirtschaftlichkeit an Geltungskraft gewinnt. Dieser Frage sind die folgenden Ausführungen gewidmet. Dabei unterscheiden wir auf der Strukturebene die institutionelle Verfasstheit der Organisation von ihren Führungs- und Qualifikationsstrukturen. Konkret prüfen wir dabei folgende Annahmen, die im Diskurs der Veränderungsdynamik eine zentrale Rolle spielen:

1. Der Siegeszug der Privaten: Der Anteil an privat geführten und profitorientierten Krankenhäusern bei den Großkrankenhäusern hat in den letzten beiden Jahrzehnten stark zugenommen (Verfasstheit der Krankenhäuser);

2. Der Trend zum managergeführten Krankenhaus: Der Anteil der Krankenhäuser, welche, analog zur Industrie, nach dem Geschäftsführer- bzw. CEO-Modell der Leitung 
organisiert sind, hat in den letzten beiden Jahrzehnten stark zugenommen (Leitungsstrukturen der Organisation);

3. Der Siegeszug der Wirtschaftswissenschaften: Der Anteil der Krankenhäuser, bei welchen die Führungskräfte u.a. betriebswirtschaftlich qualifiziert sind, hat in den letzten beiden Jahrzehnten stark zugenommen (Qualifikationsstrukturen der Organisation);

4. Die heteronome Position der Chefärztinnen und -ärzte: Die Chefarztverträge haben sich in Richtung von Angestelltenverträgen verändert und werden mit der Verpflichtung verbunden, Kriterien der Wirtschaftlichkeit besonders zu berücksichtigen (Leitungsstrukturen der Organisation).

Sollten sich diese Annahmen als zutreffend erweisen, wären auf der Strukturebene die Voraussetzungen für eine größere Geltung von Wirtschaftlichkeitskriterien geschaffen. Dies wollen wir im Folgenden prüfen.

\subsection{Stand der Forschung}

Der Literatur zufolge sind ein grundlegender Wandel der Organisation Krankenhaus und in der Folge eine De-Professionalisierung der Medizin im Krankenhaus in vollem Gange (Scott, Ruef, Mendel \& Caronna, 2000; Dent, 2003; Braun, 2009; Glassner, Pernicka \& Dittmar, 2015). Das gilt gleichermaßen für Deutschland (ebd., S. 33ff.) wie für Österreich (Flecker, Krenn \& Techernitz, 2014) und die Schweiz (Gemperle, 2014). Die deutschsprachige Diskussion nutzt dabei unterschiedliche Begriffe und Konzepte, so wird von „Verbetriebswirtschaftlichung“ (Bär, 2011), von „Industrialisierung“ (Vera, 2009), einem möglichen „Archetypenwechsel“ (Iseringhausen \& Staender, 2012), von „Mutation“ (Bode \& Vogd, 2016) oder eben von „Ökonomisierung“ (Kühn, 2008) gesprochen, wobei die insgesamt uneinheitlich verwendete These der Ökonomisierung im Diskurs sicherlich seit Jahren die prominenteste Rolle spielt (Simon, 2001). Folgt man dieser These, dann sind das Gesundheitswesen im Allgemeinen und die Patientenversorgung im Besonderen durch einen Wandel gekennzeichnet, hin zu mehr Leistungs- und Wettbewerbsorientierung (Braun, 2014). Infolge politischer Weichenstellungen scheint betriebswirtschaftliche Rationalität einen zunehmenden Einfluss auf das Denken und Handeln im Krankenhaus zu besitzen und es wird davon gesprochen, dass wirtschaftliche Kalküle medizinisch-pflegerische Ziele überformen (Marrs, 2007; Simon, 2016, S. 29f.). Die empirische Befundlage zur Frage der Ökonomisierung und De-Professionalisierung der Medizin ist jedoch eher noch dünn (Frisina Doetter \& Cacace, 2009). Dennoch gibt es einige wichtige Befunde, auf die wir bei der Überprüfung der Annahmen genauer eingehen.

Krankenhausbetriebswirtschafts- und -managementlehre haben sich, unter anderem ablesbar an einer stetig wachsenden Flut von Literatur, zunehmend mit der Re-Organisation der stationären Versorgung in Krankenhäusern - dem Krankenhausmanagement - beschäftigt (vgl. exemplarisch Salfeld, Hehner \& Wichels, 2009; hierzu auch Habersam, 2009). Das neue Steuerungsmodell, das zunächst die öffentliche Verwaltung adressierte (Holtkamp, 2008, vgl. hierzu Bär, 2011), ist zunehmend in alle Bereiche des öffentlichen Dienstes in allen drei Ländern diffundiert (Schultheis \& Gemperle, 2014). Empirisch untersucht werden diese komplexen Zusammenhänge mit Perspektive auf das Krankenhauswesen eher selten (vgl. als Ausnahme für den Ländervergleich Deutschland, Österreich und die Schweiz Flecker, Schultheis 
\& Vogel, 2014), sondern es wird zumeist bedingungslos vorausgesetzt, dass ehedem industrielle Produktions- und Organisationskonzepte im Krankenhaus aufgrund des Qualifikationswandels Anwendung finden (können und sollen) (Raible \& Leidl, 2004).

Die oft als selbstverständlich gedachte Übertragbarkeit von Konzepten und Strategien aus der Wirtschaft auf das Gesundheitssystem wird auch daran erkennbar, dass heute vielfach von Gesundheitswirtschaft und medizinischen Dienstleistungen gesprochen wird, wo es früher um die Sicherstellung von Daseinsvorsorge ging (vgl. bspw. Neumann, 2005; Marrs, 2008), für welche in der Hauptsache medizinisch und pflegerisch professionelle Maßstäbe leitend waren. Ganz allgemein wird in der Literatur von einer Konvergenz nationaler Gesundheitssysteme ausgegangen (Gottweis, Hable, Prainsack \& Wydra., 2004; Rothgang, 2006; Cacace, Götze, Schmid \& Rothgang, 2008; Schmid, Cacace, Götze \& Rothgang, 2010), die sich aufgrund ähnlicher Herausforderungen einstelle. Inwiefern die sozialstaatliche Rahmung dem Grenzen setzen kann (vgl. hierzu Bode, 2010), ist empirisch noch offen und auch, inwiefern die kollektiven Wissensbestände und Handlungsorientierungen diesen Wandel repräsentieren, ist derzeit noch zu wenig untersucht worden (vgl. aber Bär \& Pohlmann, 2016; Wilkesmann, 2016).

\subsection{Methoden und Forschungsdesign}

Die vorliegende Untersuchung konzentriert sich auf Großkrankenhäuser. Dieser Auswahlentscheidung liegt die Überlegung zugrunde, dass diese oft federführend in der Gestaltung des Strukturwandels waren. An ihnen müsste man, so unsere Annahme, daher zuerst ablesen können, welche Richtung die Veränderungsdynamik nimmt. Die Größe wurde dabei anhand des einschlägigen Kriteriums der Bettenzahl bestimmt (vgl. Klauber, Robra \& Schellschmidt, 2007, S. 20). Ausgewählt wurden so die Top-100 Krankenhäuser in Deutschland und - da die jeweilige Grundgesamtheit in den beiden Nachbarländern mit je etwa 300 Einrichtungen deutlich geringer ist als in Deutschland (knapp 2000 Häuser) - die jeweiligen Top-50 Krankenhäuser in Österreich und in der Schweiz. Die Daten für die empirischen Erhebungen stammen aus den über die jeweilige Krankenhausstatistik letztverfügbaren Stichjahren. ${ }^{3}$ Die Krankenhausdaten wurden durch Internetrecherche auf den jeweiligen Krankenhaushomepages verifiziert. In einem dynamischen Feld wie dem des Krankenhauswesens ist dieser Schritt unumgänglich, da die Anzahl an Fusionen, Rechtsform- und Trägerschaftsänderungen verhältnismäßig hoch ist.

Großkrankenhäuser in diesen drei Ländern zu vergleichen, eröffnet die Möglichkeit, die kulturelle und politisch induzierte institutionelle Varianz in diesen Veränderungsprozessen zu bestimmen und gleichzeitig den Sprachraum weitgehend konstant halten zu können. Auch wenn in der Gesundheitssystemforschung heute neue Systematisierungen vorgenommen werden (Rothgang, 2006, S. 299ff.), so ist gleichwohl die Länderauswahl auch durch die Zuordnung zu Wohlfahrtsstaatstypen begründbar. Deutschland und Österreich gelten hier idealtypischer Weise als konservativ/korporativ, die Schweiz als liberal (Kohl, 2000).

3 Für Deutschland und die Schweiz ist dies 2013, für Österreich 2015. Das Deutsche Krankenhaus Adressbuch (DKA, 2016), das Adressen und Detailinformationen zu Krankenhäusern, Kliniken und Reha-Einrichtungen getrennt nach Deutschland, Österreich und der Schweiz enthält, wurde in der aktuellen Version herangezogen, ebenso wie Daten über Desktop-Recherche im Jahr 2016 aktualisiert wurden. 
Die Öffnung für Markt und Wettbewerb im Gesundheitswesen gilt in Deutschland mit seinem System der G-DRG im Gegensatz zu einem Mischsystem in Österreich als eher radikal (Simon, 2013b), womit die drei ausgewählten Länder für deutlich unterschiedliche institutionelle Konfigurationen stehen.

Innerhalb der Großkrankenhäuser konzentrieren wir uns auf die Leitungspositionen, da ein entscheidender Beitrag zum Strukturwandel insbesondere auf diesen Positionen zu erwarten ist. Ausgehend von der Auswahl der Top-Krankenhäuser wurden die personenbezogenen Daten des ärztlichen und kaufmännischen Spitzenpersonals recherchiert. Die Erhebung deren personenbezogener Daten erfolgte durch internetbasierte Desktop-Research, z.T. ergänzt mit Kurzfragebogen zum CV. Das Untersuchungssample besteht aus der Personengruppe, die wir die Krankenhauselite nennen. Das sind die Inhaber der jeweiligen Spitzenpositionen als ärztliche und kaufmännische Direktorinnen und Direktoren. Auf diese Weise ergibt sich bei 200 ausgewählten Großkrankenhäusern in den drei Ländern ein personenbezogenes Untersuchungssample von theoretisch 400 Personen. Aufgrund sehr heterogener Führungsstrukturen mit Doppelpositionen und -spitzen, Alleingeschäftsführung und auch unbesetzten Positionen besteht das reale Sample aus 415 Personen, davon 208 in der ärztlichen und 207 in der kaufmännischen Spitzenposition (s. Tabelle 1).

Tabelle 1: Untersuchungssample. Krankenhauselite in Deutschland, Österreich und der Schweiz

\begin{tabular}{lcc}
\hline & $\begin{array}{c}\text { Ärztliche Direktoren } \\
\text { und Direktorinnen }\end{array}$ & $\begin{array}{c}\text { Kaufmännische Direktoren } \\
\text { und Direktorinnen }\end{array}$ \\
\hline Deutschland & 107 & 110 \\
Österreich & 50 & 50 \\
Schweiz & 51 & 47 \\
Gesamt & 208 & 207 \\
\hline
\end{tabular}

Quelle: Eigene Erhebung auf Basis des Deutschen Krankenhaus Adressbuch, 54. Ausgabe 2016. Freiburg: Rombach (s. auch Fußnote 3).

Aus den Lebensverlaufsanalysen nehmen wir lediglich wenige Indikatoren in die Untersuchung der hier interessierenden Fragstellung auf, z.B. die Managementausbildung (MBA und vergleichbar) oder andere Angaben zu den Qualifikationshintergründen. Die Strukturanalysen werden flankiert von einer Inhaltsanalyse der Empfehlungen zur Gestaltung von Chefarztverträgen und Sekundäranalysen. Aus bis dato 26 Interviews mit kaufmännischen und ärztlichen Direktorinnen und Direktoren geben wir selektiv in der Diskussion der Strukturbefunde einen Einblick zum relevanten Deutungs- und damit zum institutionalisierten geteilten Wissen, ohne jedoch im Ländervergleich die in allen acht Schritten ausgeführte Deutungsmusteranalyse (Pohlmann, Bär \& Valarini, 2014) hier darstellen zu können. Wenn wir uns bei der Analyse auf Querschnittsdaten stützen, dann blenden wir die für eine Diagnostik des Wandels notwendige historische Verlaufsdynamik nicht auf Basis von eigenen oder sekundären Daten ein, sondern anhand von Verweisen auf vorhandene Literatur zur jeweiligen Analysekategorie. 


\section{Zur institutionellen Verfasstheit und den Leitungsstrukturen in den Großkrankenhäusern: Die Veränderungsdynamik der regulativen Institutionen}

Im Folgenden soll geprüft werden, inwiefern die Weichen auf der Strukturebene der Großkrankenhäuser in Richtung einer stärkeren Geltungskraft von Wirtschaftlichkeit und Effizienz gestellt worden sind. Auf Basis dieser Weichenstellung werden heute die Arbeitsbeziehungen im Krankenhaus austariert. Ließen sich die oben dargestellten Annahmen bestätigen, so wäre auf der Strukturebene gezeigt, dass der autonomen Stellung der medizinischen Profession durch die stärkere Geltungskraft von Organisations- und Wirtschaftlichkeitskriterien Grenzen gesetzt und in der Folge die Arbeitsbeziehungen neu ausgehandelt und balanciert werden. Die Institutionalisierung von Wirtschaftlichkeitskriterien ginge dann mit einer De-Institutionalisierung der Profession und damit einer De-Professionalisierung der Medizin im Krankenhaus einher.

\subsection{Der Siegeszug der Privaten?}

In Deutschland wird schon seit längerem eine Tendenz zur Privatisierung des Krankenhauswesens konstatiert (Böhlke, Gerlinger, Mosebach, Schmucker \& Schulten, 2009). Die Eigentumsverhältnisse im Krankenhauswesen waren bisher durch eine differenzierte Trägerstruktur gekennzeichnet (Mayntz \& Rosewitz, 1988). Das galt für Deutschland, Österreich und die Schweiz gleichermaßen, auch wenn die Differenzierung nach Trägern in den drei Ländern unterschiedlich vorgenommen werden musste (Müller, 2008).

Die jeweiligen amtlichen Statistiken zeigen nun aber deutlich, dass sich die Eigentumsanteile in den letzten Jahren mehr oder weniger klar in Richtung privater Eigentümerschaft verändert haben. In Deutschland, das als das Land mit der umfangreichsten Privatisierung gilt, war früher der Hauptteil der Krankenhäuser öffentlich und etwa ein Drittel frei-gemeinnützig getragen. Heute finden sich je etwa ein Drittel privat, frei-gemeinnützig und öffentlich getragene Krankenhäuer (Statistisches Bundesamt, 2016). In Österreich und der Schweiz ist das Verhältnis privat zu öffentlich in etwa je 50:50 (Papouschek, 2011; Pöttler, 2014; Berger, Bienlein, Schürch \& Wegmüller, 2015). Die Krankenhäuser in öffentlicher Trägerschaft sind auch nicht länger Regiebetriebe, als Teil der öffentlichen Verwaltung geführt, sondern rechtlich selbständige Häuser, eine heterogene Mischung von Anstalten des öffentlichen Rechts, GmbHs und gemeinnützigen GmbHs etc.

Gleichwohl ist, bezogen auf die Top-100-Krankenhäuser, die Tendenz zur Privatisierung in Deutschland nicht so eindeutig, wie es bei Betrachtung aller knapp 2.000 Einrichtungen zunächst erscheint (dort sind es 35,8 Prozent privat getragene Häuser, vgl. Statistisches Bundesamt, 2016). Nach wie vor wird der größere Teil der großen deutschen Krankenhäuser, insbesondere auch der Betten, nämlich in öffentlicher Hand getragen und organisiert (vgl. Tabelle 2). Die Anzahl der privaten Häuser hat bei den Top-100-Einrichtungen zwar zugenommen, aber zumeist sind dies kleinere und mittelgroße Häuser. 
Tabelle 2: $\quad$ Struktur der Großkrankenhäuser in Deutschland, Österreich und der Schweiz nach Trägerschaft, Einrichtungen und Betten jeweils in Anzahl und Anteil

\begin{tabular}{|c|c|c|c|c|c|c|}
\hline & \multicolumn{2}{|c|}{ Öffentlich ${ }^{a}$} & \multicolumn{2}{|c|}{ Frei-gemeinnützig } & \multicolumn{2}{|c|}{ Privat } \\
\hline & Einrichtungen & Betten & Einrichtungen & Betten & Einrichtungen & Betten \\
\hline Deutschland & 73 & 92.502 & 11 & 10.478 & $16^{\mathrm{b}}$ & 15.633 \\
\hline$N=100$ & $73 \%$ & $77,9 \%$ & $11 \%$ & $8,8 \%$ & $16 \%$ & $13,2 \%$ \\
\hline Österreich & 39 & 27.392 & 5 & 1901 & 6 & 3.017 \\
\hline $\mathrm{N}=50$ & $78 \%$ & $84,8 \%$ & $10 \%$ & $5,9 \%$ & $12 \%$ & $9,3 \%$ \\
\hline Schweiz & 27 & 7.236 & 2 & 375 & 21 & 10.446 \\
\hline $\mathrm{N}=50$ & $54 \%$ & $40,0 \%$ & $4 \%$ & $2,1 \%$ & $42 \%$ & $57,8 \%$ \\
\hline
\end{tabular}

a Hier sind öffentlich getragene Häuser in öffentlich-rechtlicher Form, rechtlich selbständig wie unselbständig sowie in privatrechtlicher Form (z.B. gGmbHs) zusammengefasst.

b Die Klinika Marburg und Gießen wurden gesondert gezählt.

Quellen: Für Deutschland Daten des Statistischen Bundesamtes, für Österreich vom Bundesministerium für Gesundheit, jeweils ergänzt um das Merkmal der Trägerschaft aus dem DKA 2016, Angaben für die Schweiz wurden von der Sektion Datenmanagement und Statistik des Bundesamtes für Gesundheit zur Verfügung gestellt.

Die für Deutschland zu konstatierende Privatisierungstendenz ist in der Literatur für Österreich und die Schweiz in ähnlicher Weise beschrieben worden (für Österreich: Papouschek, 2011, S. 15; für die Schweiz: Berger et al., 2015, S. 395). In Österreich setzt dieser Wandel aber später ein (Hermann, Lindner \& Papouschek, 2009, S. 55). Erst heute lassen sich in Österreich und Deutschland ganz ähnliche Verteilungen erkennen (vgl. Tabelle 2). In der Schweiz spitzt sich diese Tendenz zur Privatisierung aber noch zu. Hier sind zwar ebenfalls über 50 Prozent der Top-50-Einrichtungen noch öffentlich getragen, jedoch mit Blick auf die zur Verfügung gestellten Bettenkapazitäten dominiert die private Trägerschaft eindeutig.

\subsection{Die Veränderung der Vorstandsstrukturen - Auf dem Weg zu managergeführten Großkrankenhäusern?}

Eine offene Frage im Diskurs um die Veränderung der strukturellen Führungskonstellationen in Großkrankenhäusern ist, inwiefern sich die Vorstands- oder Direktoriumsstrukturen in Richtung eines Geschäftsführermodells gewandelt haben. Dann hätte nach dem CEOPrinzip das Top-Management das Sagen.

Die Struktur von Krankenhausvorständen war in Deutschland noch drei Dekaden zuvor entlang der berufsständischen Versäulung dreigliedrig besetzt: mit einer ärztlichen, einer kaufmännischen und einer pflegerischen Direktionsposition (Eichhorn, 1976, S. 52f.; Krauss, 1998, S. 24f.). Doch inwieweit hat sich dies bis heute gehalten? Wir sehen für Deutschland auch hier die angenommene Änderungsdynamik, aber in sehr moderater Form. Nur in einem Viertel der untersuchten Krankenhäuser findet sich ein Geschäftsführermodell und in etwa 15 Prozent der Krankenhäuser findet sich eine Doppelstruktur der Leitung mit der Reduktion auf die kaufmännische und die ärztliche Position (vgl. Tabelle 3). Wenn man die an den Universitätsklinken überwiegend zu findende mehrgliedrige Konstellation mit einer Vertretung der medizinischen Fakultäten einbezieht (welche in Tabelle 3 bei "Mehrgliedrig entlang des Säulenmodells“ erscheint), dann ist in knapp 56 Prozent der deutschen 
öffentlich getragenen Großkrankenhäuser die Führung aber immer noch traditionell berufsständisch besetzt.

Tabelle 3: Führungsstrukturen in öffentlich getragenen Krankenhäusern im Ländervergleich (2015/16)

\begin{tabular}{lccc}
\hline Führungsstruktur & Deutschland $\mathbf{N}=\mathbf{7 0}$ & Österreich $\mathbf{N = 3 9}$ & Schweiz $\mathbf{N}=\mathbf{2 7}$ \\
\hline Mehrgliedrig entlang des Säulenmodells & $23^{\mathrm{a}}$ & $8^{\mathrm{b}}$ & $10^{\mathrm{c}}$ \\
& $32,8 \%$ & $20,5 \%$ & $37,0 \%$ \\
Klassisch dreigliedrig & 16 & 30 & 1 \\
\multirow{2}{*}{ Zweigliedrig } & $22,8 \%$ & $76,9 \%$ & $3,7 \%$ \\
& 13 & $1^{\mathrm{d}}$ & 2 \\
Geschäftsführermodell & $15,6 \%$ & $2,6 \%$ & $7,4 \%$ \\
& 18 & - & 14 \\
\hline
\end{tabular}

a Grundsätzlich dreigliedrige Struktur, die in der überwiegenden Zahl der Universitätsklinika Sonderformen findet, welche zusätzlich eine Vertretung der medizinischen Fakultäten vorsehen.

b Vierköpfiges Gremium mit einer Position zusätzlich zur dreigliedrigen Struktur (techn. Direktion oder Qualitätsmanagement).

c Sechs- bis achtköpfige Gremien mit jeweils einer Vorsitzenden-Position.

d Der kollegialen Führung übergeordnetes zweiköpfiges Managementboard.

Quelle: Eigene Erhebung auf Basis des Deutschen Krankenhaus Adressbuch, 54. Ausgabe 2016. Freiburg: Rombach und Desktop-Recherche der Homepages der betreffenden Krankenhäuser.

In Österreich hingegen liegt die viel stärkere Kontinuität in den gesetzlichen Vorschriften begründet. Hier wird nach dem Krankenanstaltengesetz (KAKuG) die kollegiale Führung durch eine ärztliche Leitung, die Verwaltungsleitung und die Leitung des Pflegedienstes als Mindestbesetzung ausgeführt (Pöttler, 2014, S. 188ff.). Nimmt man die vierköpfigen Führungsgremien hinzu, bei denen in der Regel Zusatzpositionen für gesonderte spezifische Aufgaben (Technik und Qualitätsmanagement) bestehen, dann gibt es nur wenige Ausnahmen von dieser Führungsstruktur der öffentlichen Häuser.

Auch in der Schweiz war lange Zeit die Dreigliedrigkeit - häufig bezeichnet als „,Dreibein“ (Endrissat \& Müller, 2007) - mit parallelen Hierarchien und einer entsprechenden Abbildung in der Spitalleitung charakteristisch (Boos, 2004). Hier ist mittlerweile aber das CEO-Prinzip im Falle der privaten Klinken zum Standard geworden (Berger et al., 2015, S. 397). In über der Hälfte der öffentlichen Einrichtungen dominiert ebenfalls das Geschäftsführermodell (vgl. Tabelle 3). Die berufsständische Dreier-Struktur ist damit hier nahezu abgelöst. Die angenommene Änderungsdynamik schlägt sich hier am drastischsten nieder.

\subsection{Der Siegeszug der Wirtschaftswissenschaften?}

Das Juristenmonopol in der Krankenhausverwaltung ist Geschichte. Hatten noch drei Dekaden zuvor regelmäßig Personen mit Jurastudium die Stellen in der Spitze der Verwaltung von deutschen Großkrankenhäusern inne, so sind daraus heute kaufmännische Direktorenbzw. Direktorinnenstellen geworden, die überwiegend mit Kaufleuten besetzt sind. Der Wechsel von der Verwaltung zum Management gilt in Bezug auf diese Positionen daher formal als vollzogen (Franke, 2007, S. 34ff.; Baumann, 2008, S. 42; Salfeld, Hehner \& Wi- 
chels, 2009, S. 27ff.; Blum, Löffert, Offermanns \& Steffen, 2015). Dies ist heute bei den Großkrankenhäusern in Deutschland überwiegend der Fall (vgl. Tabelle 4; vgl. hierzu auch Bär, 2011; Krieg, 2015). Fragt man darüber hinaus nach den wirtschaftswissenschaftlichen Zusatzqualifikationen der ärztlichen Direktorinnen und Direktoren, so sieht man in Deutschland noch eher geringe Anteile. Doch vieles deutet in unseren Fallstudien darauf hin, dass diese in der nächsten Generation medizinischer Führungskräfte weiter zunehmen werden.

Bei den Schweizer kaufmännischen Direktorinnen und Direktoren haben etwa 40 Prozent nach ihrer grundständigen Qualifikation - für über die Hälfte ist dies eine wirtschaftswissenschaftliche, sie reicht jedoch sehr heterogen von Politikwissenschaft über Rechtswissenschaften bis zu Naturwissenschaften - noch einen MBA-Abschluss erworben. Auch in Österreich ist das Bild vergleichbar. Nach einem in der Regel spezifisch krankenhausbetriebswirtschaftlichen Studium haben viele noch einen dem MBA vergleichbaren Abschluss, nach einem Rechts- oder einem anderen Studium haben sogar die meisten einen MBA erworben. In der Summe sind es knapp 40 Prozent in der kaufmännischen Führungsposition in Österreich. Dem gegenüber hat dieser Zusatzabschluss in Deutschland lediglich einen geringen Stellenwert, hier besitzen lediglich 12,7 Prozent der kaufmännischen Direktorinnen oder Direktoren einen MBA oder vergleichbaren Abschluss (vgl. Tabelle 4).

In der Schweiz und in Österreich hingegen verfügen vergleichbar mit den kaufmännischen auch viele von den ärztlichen Leitungen über eine solche Zusatzqualifikation (jew. 40 und mehr Prozent, vgl. Tabelle 4). In Österreich finden sich dabei ganz unterschiedliche Zusatzqualifikationen mit wirtschaftswissenschaftlicher Komponente, es handelt sich hierbei um postgraduale Abschlüsse wie Gesundheitsmanagement und Public Health, Management für Health Professionals oder den spezifischen Abschluss als akademisch geprüfter Krankenhausdirektor (vgl. auch Diem \& Dorner, 2014).

Tabelle 4: Wirtschaftswissenschaftliche (Zusatz-)Qualifikationen in der Krankenhauselite in Deutschland, Österreich und der Schweiz

\begin{tabular}{lccc}
\hline & $\begin{array}{c}\text { Ärztliche Direktoren/ } \\
\text { Direktorinnen mit MBA } \\
\text { oder vergleichbar }\end{array}$ & $\begin{array}{c}\text { Kaufmännische Direktoren/ } \\
\text { Direktorinnen mit wirtschaftswis- } \\
\text { senschaftlicher Qualifikation }\end{array}$ & $\begin{array}{c}\text { Kaufmännische Direktoren/ } \\
\text { Direktorinnen mit } \\
\text { Zusatzqualifikationen, MBA } \\
\text { oder vergleichbar }\end{array}$ \\
\hline Deutschland & $19,6 \%$ & $60,7 \%$ & $12,7 \%$ \\
Österreich & $40,0 \%$ & $60,0 \%$ & $38,0 \%$ \\
Schweiz & $42,1 \%$ & $53,0 \%$ & $47,0 \%$ \\
\hline
\end{tabular}

Quelle: Eigene Erhebung auf Basis Deutschen Krankenhaus Adressbuch, 54. Ausgabe 2016. Freiburg: Rombach und Desktop-Recherche der Homepages der entsprechenden Krankenhäuser.

Kaufmännische Führungskräfte verfügen heute in den großen Krankenhäusern also überwiegend über wirtschaftswissenschaftliche Qualifikationen, in der Schweiz und Österreich zudem über postgraduale Abschlüsse wie den MBA oder vergleichbare. Die Annahme des Siegeszugs der Wirtschaftswissenschaften lässt sich mit kleineren Abstrichen in der Tendenz also bestätigen. Es zeigt sich darüber hinaus, dass eine ökonomische (Zusatz-)Qualifikation bei der ärztlichen Leitung in den Führungsgremien deutscher Krankenhäuser eine 
Rolle spielt, aber dass die Anteile noch begrenzt bleiben, während in Österreich und der Schweiz ein größerer Anteil darüber verfügt.

\subsection{Die heteronome Position der Chefärztinnen und Chefärzte? - Die Chefarztverträge}

Mit Blick auf die Arbeitsbeziehungen und den Status der Medizinerinnen und Mediziner wurde die Annahme formuliert, dass sie an der Spitze der Hierarchie zu leitenden Angestellten und in den Stufen darunter zu Fachkräften - ,,von Fürsten zu Knechten“ (Wilkesmann, 2016) - degradiert würden. Ihre fachliche Expertise erbrächten Ärztinnen und Ärzte im klinischen Geschehen heute als medizinische Dienstleistung in einem entlang von betrieblichen Prozessen gemanagten und reorganisierten Krankenhaus. Die Begrenzung der Autonomie in der Arzt-, wie der Chefarztposition durch ökonomische Zwänge gilt als eine der zentralen Dimensionen des aktuellen Wandels (Siegrist, 2012). Gegenwehr im Sinne der Veränderungen der Arbeitsbeziehungen sind u.a. die Etablierung des Marburger Bundes als Berufsgewerkschaft ${ }^{4}$ und seit Jahren regelmäßig wiederkehrende Streiks der Ärzteschaft (Bär, 2010; Greef, 2012; Pichelbaur, 2014; Martinz, 2015).

Die vertragliche Gestaltung der Arbeitsbeziehungen erfährt dabei besondere Aufmerksamkeit. Hier werde die „Degradierung“ der Chefärztinnen und -ärzte zu leitenden Angestellten, die auch auf die Verfolgung ökonomischer Zwecke verpflichtet werden, manifest. So bezeichnet eine Stellungnahme der Bundesärztekammer (BÄK) Neuerungen in den Empfehlungen als „Überregulierung der Pflichten von Chefärzten“ und erkennbare „Aushöhlung jeglichen freiberuflichen Elements chefärztlicher Tätigkeit“ (BÄK, 2011). „Berufspolitisch und berufsrechtlich völlig inakzeptabel“" (ebd.) war das Statement der Vorstände der BÄK, des Verbandes der leitenden Krankenhausärzte Deutschlands und des Marburger Bundes zu dieser Frage.

Dabei zeichnen sich in den Chefarztverträgen ${ }^{5}$ derzeit zwei zentrale Entwicklungen ab: Chefärztinnen und -ärzte werden tatsächlich stärker auf die Beachtung wirtschaftlicher Faktoren verpflichtet und vertraglich zunehmend als leitende Angestellte geführt. Die Vergütungssysteme in den drei Ländern sind sehr unterschiedlich (KPMG, 2011). Für Deutschland ist in den Großkrankenhäusern davon auszugehen, dass bei Neuverträgen die Empfehlungen der Deutschen Krankenhausgesellschaft (DKG) zur Gestaltung von Chefarztverträgen Anwendung finden (Blum, Offermanns \& Perner, 2008). In Österreich sind die ärztlichen Vergütungen durch Spitalgesetze und Besoldungsvorschriften der Bundesländer geregelt. Leitende Ärztinnen und Ärzte können privat liquidieren (KPMG, 2011). In der Schweiz kommen für Chefarztverträge überwiegend nicht die gesetzlichen Regelungen zum Tragen (ebd.). Auf der einen Seite versucht die Ärzteseite in der Schweiz durch Positionspapiere normative Grundlagen für die vertraglichen Verhandlungen bei bspw. Bonusregelungen zu geben (Meyer, 2013), auf der anderen Seite (Schweizerische Sanitätsdirektorenkonferenz, 2012) wird versucht, die Chefarztverträge stärker in Richtung Leistungs- und Ergebnisorientierung hin zu gestalten.

4 Oder in Österreich die Gründung von Asklepios.

5 Analysiert wurden ausschließlich die Vertragsempfehlungen der DKG, also lediglich der deutsche Fall, da ähnliche Dokumente für Österreich und die Schweiz nicht vorlagen und nicht bekannt sind. 
Bereits 1989/90 wurden Chefärztinnen und -ärzte in Deutschland durch die DKGEmpfehlungen dazu verpflichtet, wirtschaftliche Grundsätze zu beachten. Chefarztverträge nach den Neuerungen seit 2002 (Baur, 2002) sehen dann für sie die ökonomische Mitverantwortung für den Krankenhausbetrieb vor. Hinsichtlich der Patientenversorgung ist der Chefarzt bzw. die Chefärztin nicht mehr nur zum ,zweckmäßigen, wirtschaftlichen und sparsamen“ Umgang mit den zur Verfügung stehenden Mitteln des Krankenhauses verpflichtet, sondern wird auch für den entsprechenden Mitteleinsatz durch nachgeordnete Ärztinnen und Ärzte und die anderen Mitarbeitenden in der Abteilung verantwortlich gemacht. Neu ist auch die Umstellung der Regelungen für die Privatambulanz und liquidation. Künftig können Chefärztinnen bzw. -ärzte die inhaltliche Ausrichtung des privaten Ambulanzbetriebes nicht mehr als Unternehmen im Unternehmen unabhängig vom Krankenhaus gestalten. Die ambulante Tätigkeit wird nun in den Dienstaufgabenkatalog integriert. Dies bedeutet, dass die private Liquidation der ambulanten Tätigkeit entfällt. Chefärztinnen und -ärzte mussten früher einen Teil der Erlöse an das Krankenhaus abführen. Nun ist es umgekehrt. Sie werden vom Krankenhaus an den Erlösen der Leistungen, welche nun Krankenhausleistungen darstellen, beteiligt. Damit entfällt die Option, die Privatambulanz unabhängig vom Krankenhausbetrieb mit einem eigenen inhaltlichen Schwerpunkt zu führen. Ebenso verhält es sich mit der Privatliquidation als bisher weitaus höherem Verdienstanteil an der Gesamtvergütung. Alle erbrachten Leistungen sind nun Krankenhausleistungen, unabhängig davon, ob sie durch das oder im Krankenhaus erbracht worden sind. Alternativ werden dafür noch Muster für Nebentätigkeitserlaubnis und Nutzungsvertrag angeboten.

Der Blick auf die ab 2007 gültigen Musterverträge (DKG, 2007) zeigt zur weiteren ökonomischen Einbindung zunächst keine Neuerungen ${ }^{6}$. Allerdings wird durch die Aufnahmeempfehlung eines sogenannten Versetzungsvorbehalts in Anmerkung 5 (ebd., S. 16f.) - welcher bedeutet, dass im Bedarfsfall, nach Maßgabe des Trägers, wenn bspw. in einer Kette mehrere Krankenhäuser betrieben werden, der betreffende Chefarzt bzw. die betreffende Chefärztin in ein anderes Haus versetzt werden kann - deutlich, dass es um die Autonomie in der Chefarztposition nun nicht mehr gut bestellt ist. Wie jedes andere Personal auch kann die Chefärztin bzw. der Chefarzt nun offenbar zu den frei verfügbaren $\mathrm{Hu}$ manressourcen gezählt werden und wird disponibel und austauschbar. Die symbolische Bedeutung dessen ist nicht zu unterschätzen, weist sie doch darauf hin, dass es sich bei Chefärztinnen und -ärzten um Personal handelt, über das verfügt werden kann.

Insgesamt lässt sich im Vergleich feststellen, dass es der Annahme entsprechend zu einer immer weiteren Einbindung von Chefärztinnen und -ärzten in die ökonomische Verantwortung für das Krankenhaus gekommen ist. Dies geschah nicht aus, sondern gerade gegen die Interessen der Ärzteschaft, welche ökonomische Fragen und Probleme, so könnte man sagen, gerne weiterhin aus dem Bereich der Medizin externalisiert gehalten hätte. Ärztinnen und Ärzte sowie ihre Vertreter sahen und sehen dadurch nämlich wesentliche Elemente der Freiberuflichkeit ärztlicher Tätigkeit im Schwinden (BÄK, 2003, S. 1633).

6 In 2013 wurden diese im Detail nochmals aktualisiert, um die in die Kritik geratenen leistungsbezogenen Zielvereinbarungen zu entschärfen (B ̈KK, 2013), jedoch nicht grundsätzlich neu gefasst. 


\section{$4 \quad$ Diskussion und Fazit}

Man kann zusammenfassend festhalten, dass die institutionellen Weichen im Krankenhauswesen bei den öffentlichen Krankenhäusern in Richtung einer stärkeren Geltung von Wirtschaftlichkeitskriterien gestellt sind. Pacemaker in diesem Prozess sind die Schweizer Großkrankenhäuser. Das liberale Wohlfahrtsstaatsmodell der Schweiz schlägt sich auch im Krankenhausmanagement nieder. In allen herangezogenen Weichenstellungen auf der Strukturebene sind jene in der Schweiz am weitreichendsten in Richtung Ökonomisierung gestellt. Alle geprüften Annahmen bestätigen sich weitgehend. Der Siegeszug der Privaten lässt sich an der Dominanz der privat geführten Großkrankenhäuser klar ablesen. Das Geschäftsführermodell ist weit verbreitet und der Siegeszug der Wirtschaftswissenschaften bestätigt sich. Auch wird nach bisherigen Informationen versucht, die Chefarztverträge in Richtung einer stärkeren Leistungs- und Ergebnisorientierung hin $\mathrm{zu}$ gestalten. Für Deutschland bestätigen sich viele Annahmen auch. Dennoch zeigt sich für die Großkrankenhäuser ein gemischtes Bild. Zwar hat der Anteil an privat geführten und profitorientierten Krankenhäusern bei den Großkrankenhäusern in den letzten beiden Jahrzehnten zugenommen, aber eine öffentliche Trägerschaft dominiert nach wie vor. Auch vom Trend zum managergeführten Krankenhaus lässt sich bei den Großkrankenhäusern noch nicht sprechen, da der Anteil der Krankenhäuser, welche analog zur Industrie, nach dem Geschäftsführer- bzw. CEO-Modell der Leitung organisiert sind, nur bei rund 15 Prozent liegt. Weitreichender ist der Siegeszug der Wirtschaftswissenschaften in Deutschland. Der Anteil der Krankenhäuser, bei welchen die Führungskräfte unter anderem betriebswirtschaftlich qualifiziert sind, hat in den letzten beiden Jahrzehnten zugenommen, und das Juristenmonopol der Verwaltungsdirektion gehört endgültig der Vergangenheit an. Gemessen an der Entwicklung der Chefarztverträge lässt sich auch eine zunehmend heteronome Position der Chefärztinnen und -ärzte erkennen. In der Tat haben sich die Chefarztverträge in Richtung von Angestelltenverträgen verändert und werden nun mit der Verpflichtung verbunden, Kriterien der Wirtschaftlichkeit besonders zu berücksichtigen.

Tabelle 5: Übersicht über die zentralen Befunde

\begin{tabular}{llll}
\hline $\begin{array}{l}\text { Dimensionen des } \\
\text { Strukturwandels }\end{array}$ & Deutschland & Österreich & Schweiz \\
\hline $\begin{array}{l}\text { Privat getragene } \\
\text { Krankenhäuser? }\end{array}$ & $\begin{array}{l}\text { Zunahme, aber Dominanz } \\
\text { Öffentlicher Trägerschaften }\end{array}$ & $\begin{array}{l}\text { Leichte Zunahme, aber Domi- } \\
\text { nanz öffentlicher Trägerschaften }\end{array}$ & $\begin{array}{l}\text { Starke Zunahme, Dominanz } \\
\text { privat getragener Häuser }\end{array}$ \\
$\begin{array}{l}\text { Geschäftsführer- bzw. Zunahme, aber Dominanz drei- } \\
\text { CEO-Modell? }\end{array}$ & $\begin{array}{l}\text { Dominanz dreigliedriger Lei- } \\
\text { gliedriger Leitungsstrukturen }\end{array}$ & $\begin{array}{l}\text { Dominanz des Geschäftsführer- } \\
\text { tungstrukturen }\end{array}$ \\
$\begin{array}{l}\text { Betriebswirtschaftli- } \\
\text { che Qualifikationen? }\end{array}$ & $\begin{array}{l}\text { Dominant in der kaufmänni- } \\
\text { schen Direktion, }\end{array}$ & $\begin{array}{l}\text { Dominant in der kaufmänni- } \\
\text { schen Direktion, }\end{array}$ & $\begin{array}{l}\text { Dominant in der kaufmänni- } \\
\text { schen Direktion, }\end{array}$ \\
& $\begin{array}{l}\text { Zunehmende Bedeutung als Zu- } \\
\text { satz in der ärztlichen Direktion }\end{array}$ & $\begin{array}{l}\text { Zunehmende Bedeutung in der } \\
\text { ärztlichen Direktion + MBA }\end{array}$ & $\begin{array}{l}\text { Zunehmende Bedeutung in der } \\
\text { ärztlichen Direktion + MBA }\end{array}$ \\
$\begin{array}{ll}\text { Chefärztinnen und } \\
\text {-ärzte als leitende } \\
\text { Angestellte? }\end{array}$ & $\begin{array}{l}\text { Radikaler Wandel bei Chefarzt- } \\
\text { verträgen }\end{array}$ & $\begin{array}{l}\text { Besoldungsvorschriften + } \\
\text { Privatliquidationen }\end{array}$ & $\begin{array}{l}\text { Gestaltung in Richtung stärkerer } \\
\text { Leistungs- und Ergebnisorientie- } \\
\text { rung }\end{array}$ \\
\hline
\end{tabular}

Quelle: Eigene Darstellung 
In Österreich mit seinem konservativ-korporativen Wohlfahrtsstaatsmodell fallen diese Weichenstellungen in Richtung Ökonomisierung noch moderater als in Deutschland aus. Die Dominanz öffentlich-rechtlicher Trägerschaft ist mit 78 Prozent der Top-50 Krankenhäuser noch stärker als in Deutschland und die Dreigliedrigkeit in den Leitungsstrukturen ist rechtlich vorgegeben. Allerdings ist der Siegeszug der Wirtschaftswissenschaften hier noch etwas stärker als in Deutschland und die leitenden Ärztinnen und Ärzte dürfen in der Regel noch privat liquidieren.

Alle geprüften Dimensionen des Strukturwandels zeigen damit, dass die Strukturkonservativität der Krankenhäuser in den drei Ländern durchbrochen ist. Es zeichnet sich ein Wandel ab, der sich in Deutschland und in Österreich sowohl in den Bahnen der institutionellen Ordnungen, als auch im Rahmen der institutionell verfestigten Pfade vollzieht. Nur die Schweiz hat diesen Pfad erkennbar seit längerem verlassen. Sie ist in der ökonomischen Modernisierung der Großkrankenhäuser nach dem Vorbild moderner Wirtschaftsunternehmen vorausgeeilt. Doch auch für Deutschland und Österreich gilt: Es ist überraschend, dass sich in allen herangezogenen Strukturdimensionen ein ähnlicher Wandel in Richtung einer strukturellen Aufwertung der betriebswirtschaftlichen Seite der Großkrankenhäuser vollzieht. Er lässt sich zwar für Deutschland und Österreich nicht so verstehen, dass aus öffentlich getragenen gemeinwohlorientierten Großkrankenhäusern profitorientierte Unternehmen werden - das ist unseren Ergebnissen zufolge weit gefehlt. Aber eine Aufwertung der Legitimität betriebswirtschaftlicher Effizienzgedanken lässt sich an den Strukturen der Großkrankenhäuser ablesen. Diese Formalstrukturen und formalen Weichenstellungen zeigen an, dass die Profession der Medizin in den Großkrankenhäusern derzeit eine Organisierung erfährt, welche die Geltungschancen wirtschaftlicher Ansprüche erhöht. Dieser Prozess, der auch die Arbeitsbeziehungen maßgeblich verändert, lässt sich nicht einfach als Autonomieverlust der Medizin charakterisieren, sondern viel besser als zunehmende betriebswirtschaftliche Rahmung in der Ausübung ihres Mandats zur autonomen Problembearbeitung. Der fachliche Kern der Krankenbehandlung des einzelnen Patienten wird dadurch nur wenig tangiert, aber die dienstleistungsorientierte Behandlung des Patientenkollektivs wird stärker an die Notwendigkeiten betriebswirtschaftlicher Effizienz und politischer Regulierung angepasst.

Aber dieser Artikel blieb auf die institutionellen Weichenstellungen durch die regulativen Institutionen in den drei Ländern beschränkt. Erst ihr Zusammenspiel mit den kognitiven und normativen Institutionen in diesem Feld kann letztlich zeigen, wie sie sich konkret umsetzen. Dazu haben wir für Österreich und die Schweiz noch keine belastbaren Befunde. Für Deutschland aber deutet sich in unseren Analysen an, dass diese institutionellen Weichenstellungen den Direktorinnen und Direktoren in den Großkrankenhäusern nicht äußerlich bleiben. Auch in der Medizin wird bei den derzeit amtierenden Interviewpartnern nun eine gleichberechtigte Geltung betriebswirtschaftlicher Leitungsansprüche akzeptiert, was die älteren Kohorten in unserem Sample noch abgelehnt hatten. Doch die Kampflinie scheint nun anders zu verlaufen. Es handelt sich nicht mehr um Rückzugsgefechte der Ärzteschaft im Krankenhaus, sondern um professionelle Vereinnahmungsstrategien des betriebswirtschaftlichen Kompetenzfeldes durch diese. Mit ein bisschen Training, so die Devise, kann die medizinische Leitung das ohnehin besser. Die rahmensetzende Machtfülle der kaufmännischen Direktion könnte auf diese Weise elegant in jene der betriebswirt- 
schaftlich fortgebildeten ärztlichen Führungskraft überführt werden, insoweit es dieser gelingt, die Geschäftsführer- bzw. Vorstandspositionen zu besetzen. Immer mehr jedenfalls wird dieser Konflikt in den deutschen Großkrankenhäusern nach unseren Ergebnissen nun auf Augenhöhe ausgetragen, und immer weniger sind die Strukturen geeignet, einen absoluten Hoheitsanspruch der Medizin im Großkrankenhaus zu unterstützen. Die kognitive Institution der medizinischen Veranstaltung Krankenhaus mit klarer Orientierung an medizinischen Belangen, wie sie durch die Profession der Medizin vorgegeben ist, hat gleichwohl unhinterfragt Bestand.

\section{Literatur}

Abbott, A. (1988). The System of Professions. An Essay on the Divison of Expert Labour. Chicago: University Press.

Bär, S., \& Pohlmann, M. (2016). Kurswechsel im Krankenhaus. Auf dem Weg zu einer markt- und profitorientierten Dienstleistungsorganisation? In I. Bode, \& W. Vogd (Hrsg.), Mutationen des Krankenhauses. Soziologische Diagnosen in organisations- und gesellschaftstheoretischer Perspektive (S. 229-250). Wiesbaden: Springer VS. doi:10.1007/978-3-658-11853-2_12

Bär, S. (2010). Kämpfe um die Autonomie der Medizin. Überlegungen zu Arbeitskämpfen im Krankenhaus. In G. Schweiger, \& B. Brandl (Hrsg.), Der Kampf um Arbeit. Dimensionen und Perspektiven (277-310). Wiesbaden: VS Verlag für Sozialwissenschaften. doi:10.1007/978-3-531-92505-9_10

Bär, S. (2011). Das Krankenhaus zwischen ökonomischer und medizinischer Vernunft. Krankenhausmanager und ihre Konzepte. Wiesbaden: VS Verlag für Sozialwissenschaften. doi:10.1007/978-3-531-93349-8_4

Baumann, H. (2008). Krankenhausbetriebswirtschaftslehre als spezielle Betriebswirtschaftslehre. In: W. Hellmann, H. Baumann, M. L. Bienert, \& D. P. Wichelhaus (Hrsg.), Krankenhausmanagement für Leitende Ärzte (S. 32-51). Heidelberg, München, Landsberg, Berlin: medhochzwei Verlag.

Baur, U. (2002). Mustervertrag mit Arbeitgeberschlagseite. Deutsches Ärzteblatt, 99, 1495-1497.

Berger, S., Bienlein, M., Schürch, D., \& Wegmüller, B. (2015). Spitäler. In W. Oggier (Hrsg.), Gesundheitswesen Schweiz 2015-2017 (5. vollständig überarbeitete Aufl.) (S. 393-411). Bern: Hans Huber Verlag.

Blum, K., Löffert, S., Offermanns, M., \& Steffen, P. (2015). Krankenhausbarometer. Umfrage 2015. Deutsches Krankenhausinstitut. Düsseldorf.

Blum, K., Offermanns, M., \& Perner, P. (2008). Krankenhausbarometer. Umfrage 2008. Deutsches Krankenhausinstitut. Düsseldorf.

Bode, I., \& Vogd, W. (Hrsg.) (2016). Mutationen des Krankenhauses. Soziologische Diagnosen in organisations- und gesellschaftstheoretischer Perspektive. Wiesbaden: Springer VS. doi:10.1007/978-3-658-11853-2

Bode, I. (2010). Der Zweck heil(ig)t die Mittel? Ökonomisierung und Organisationsdynamik im Krankenhaussektor. In M. Endreß, \& T. Matys (Hrsg.), Die Ökonomie der Organisation - die Organisation der Ökonomie (S. 63-92). Wiesbaden: VS Verlag für Sozialwissenschaften. doi:10.1007/978-3-531-92119-8_4

Böhlke, N., Gerlinger, T., Mosebach, K., Schmucker, R., \& Schulten, T. (Hrsg.). (2009). Privatisierung von Krankenhäusern. Erfahrungen und Perspektiven aus Sicht der Beschäftigten. Hamburg: VSA Verlag.

Boos, L. (2004). Soziales Dilemma und die Organisation des Krankenhauses. Die Aufgaben des Spitalmanagements. Bern: Schweizerische Gesellschaft für Gesundheitspolitik. 
Braun, B. (2009). Krankenhaus unter DRG-Bedingungen: Zwischen Ökonomisierung, Unwirtschaftlichkeit, Veränderungsresistenz und Desorganisation. In R. Böckmann (Hrsg.), Gesundheitsversorgung zwischen Solidarität und Wettbewerb (S. 117-139). Wiesbaden: VS Verlag für Sozialwissenschaften. doi:10.1007/978-3-531-91419-0_6

Braun, B. (2014). Auswirkungen der DRGs auf Versorgungsqualität und Arbeitsbedingungen im Krankenhaus. In A. Manzei, \& R. Schmiede (Hrsg.), 20 Jahre Wettbewerb im Gesundheitswesen. Theoretische und empirische Analysen zur Ökonomisierung von Medizin und Pflege (S. 91113). Wiesbaden: Springer VS. doi:10.1007/978-3-658-02702-5_4

Bundesärztekammer (B̈̈K) (2003). Bekanntmachungen. Gemeinsame Hinweise der Bundesärztekammer, des Verbandes der leitenden Krankenhausärzte Deutschlands und des Marburger Bundes zu den Grundpositionen und -regelungen der „Beratungs- und Formulierungshilfe Chefarzt-Vertrag“ der Deutschen Krankenhausgesellschaft. Deutsches Ärzteblatt, 100, 16331635 .

Bundesärztekammer (BÄK) (2011). DKG-Chefarztvertragsmuster - Abkehr von medizinischen Prioritäten und Ausrichtung auf ökonomische Vorgaben. Abgerufen von http://www.bundesaerztekammer.de/ page.asp?his=1.144.761.1039

Bundesärztekammer (BÄK) (2013). Empfehlungen zu leistungsbezogenen Zielvereinbarungen in Chefarztverträgen vorgelegt. Abgerufen von http://www.bundesaerztekammer.de/page.asp?his=3.71.11025.11227.11237

Cacace, M., Götze, R., Schmid, A., \& Rothgang, H. (2008). Explaining Convergence and Common Trends in the Role of the State in OECD Health Care System. Harvard Health Policy Review, 9 (1), 5-16.

Dent, M. (2003). Remodelling Hospitals and Health Professions in Europe. Medicine, Nursing and the State. Basingstoke/New York: Palgrave Macmillan. doi:10.1057/9781403938411

Deutsche Krankenhausgesellschaft (DKG) (2007). Beratungs- und Formulierungshilfe Chefarztvertrag (8. Aufl.) 2007. Düsseldorf: DKVG.

Diem, G., \& Dorner, T. E. (2014). Public Health Ausbildung in Österreich. Ein Überblick. Wiener Medizinische Wochenschrift 164, 131-140. doi:10.1007/s10354-014-0278-9

DKA (2016). Deutsches Krankenhaus Adressbuch, 54. Ausgabe 2016. Freiburg: Rombach Druckund Verlagshaus.

Eichhorn, S. (1976). Krankenhausbetriebslehre. Theorie und Praxis des Krankenhausbetriebes Band II. Stuttgart: Kohlhammer.

Endrissat, N., \& Müller, W. R. (2007). Führung in Spitälern - Führungsselbstverständnisse von Managern und Medizinern im Vergleich. Wirtschaftswissenschaftliches Zentrum (WWZ) der Universität Basel. Forschungsbericht 06/07 N.

Flecker, J., Krenn, M., \& Techernitz, M. (2014). Der dreifache Bezug zur Arbeit - Transformation öffentlicher Dienste und berufliche Identität. Österreichische Zeitschrift für Soziologie, 39, 199219. doi:10.1007/s11614-014-0138-2

Flecker, J., Schultheis, F., \& Vogel, B. (Hrsg.). (2014). Im Dienste öffentlicher Güter. Metamorphosen der Arbeit aus der Sicht der Beschäftigten. Berlin: edition sigma.

Franke, D. H. (2007). Krankenhaus-Management im Umbruch. Konzepte - Methoden - Projekte. Stuttgart: Kohlhammer

Frisina Doetter, L., \& Cacace, M. (2009). DRGs and the Professional Independence of Physicians. In A.N. Dwivedi (Hrsg.), Handbook of Research on Information Technology Management and Clinical Data Administration in Healthcare (173-191). Hershey, PA: IGI Global. doi:10.4018/9781605663562.ch012

Gemperle, M. (2014). „Der Patient wird nicht gesunder, wenn wir sagen, wir müssen betriebswirtschaftlich denken“. Fallstudie zum Strukturwandel der Arbeit im schweizerischen Krankenhaus- 
bereich. In J. Flecker, F. Schultheis, \& B. Vogel (Hrsg.), Im Dienste öffentlicher Güter. Metamorphosen der Arbeit aus der Sicht der Beschäftigten (S. 31-56). Berlin: edition sigma.

Gerlinger, T., \& Mosebach, K. (2009). Die Ökonomisierung des deutschen Gesundheitswesens: Ursachen, Ziele und Wirkungen wettbewerbsbasierter Kostendämpfungspolitik. In N. Böhlke, T. Gerlinger, K. Mosebach, R. Schmucker, \& T. Schulten (Hrsg.), Privatisierung von Krankenhäusern. Erfahrungen und Perspektiven aus Sicht der Beschäftigten (S. 10-40). Hamburg: VSA Verlag.

Glassner, V., Pernicka, S., \& Dittmar, N. (2015). Arbeitsbeziehungen im Krankenhaussektor. Reihe Study der Hans-Böckler-Stiftung, Band 306. Düsseldorf.

Gottweis, H., Hable, W., Prainsack, B. \& Wydra, D. (2004). Verwaltete Körper. Strategien der Gesundheitspolitik im internationalen Vergleich. Wien, Köln, Weimar: Böhlau.

Greef, S. (2012). Die Transformation des Marburger Bundes. Vom Berufsverband zur Berufsgewerkschaft. Wiesbaden: VS Verlag für Sozialwissenschaften. doi:10.1007/978-3-531-19574-2

Habersam, M. (2009). Management öffentlicher Krankenhäuser. Eine Rekonstruktion der theoretischen Grundlagen. Wiesbaden: VS Verlag für Sozialwissenschaften. doi:10.1007/978-3-531-91626-2

Hermann, C., Lindner, D., \& Papouschek, U. (2009). Privatisierung öffentlicher Dienstleistungen und Folgen für die Beschäftigung, Arbeitsbedingungen und Arbeitsbeziehungen - österreichische Ergebnisse. In AK-Wien (Hrsg.), Zur Zukunft öffentlicher Dienstleistungen (S. 51-83). Wien: FORBA.

Holtkamp, L. (2008). Das Scheitern des Neuen Steuerungsmodells. dms - der moderne Staat - Zeitschrift für Public Policy, Recht und Management, 2, 423-446.

Iseringhausen, O., \& Staender, J. (2012). Das Krankenhaus als Organisation. In M. Apelt, \& V. Tacke (Hrsg.), Handbuch Organisationstypen (S. 185-203). Wiesbaden: VS Verlag für Sozialwissenschaften. doi:10.1007/978-3-531-93312-2_10

Klatetzki, T., \& Nokielski, H. (2010). Soziale personenbezogene Dienstleistungsorganisationen als bürokratisch-professionelle Handlungszusammenhänge: Weber und die Folgen. In T. Klatetzki (Hrsg.), Soziale personenbezogene Dienstleistungsorganisationen. Soziologische Perspektiven (S. 8-24). Wiesbaden: VS Verlag für Sozialwissenschaften. doi:10.1007/978-3-531-92474-8_2

Klauber, J., Robra, B-P., \& Schellschmidt, H. (Hrsg.). (2007). Krankenhausreport 2008/2009. Stuttgart/New York: Schattauer.

Kohl, J. (2000). Der Sozialstaat: Die deutsche Version des Wohlfahrtsstaates - Überlegungen zu seiner typologischen Verortung. In S. Leibfried, \& U. Wagschal (Hrsg.), Der deutsche Sozialstaat. Bilanzen - Reformen - Perspektiven (S. 115-152). Frankfurt, New York: Campus.

KPMG (2011). Auswertung öffentlich verfügbarer Quellen zu europäischen Ärztegehältern. Abgerufen von www.dkgev.de/.../9957.2011-08-18_KPMG-

Studie_Auswertung_oeffentlich_verfuegbarer_Quellen_zu_europaeischen_Aerztegehaeltern.pdf

Krauss, R. (1998). Transformationsprozesse im Krankenhaus. München und Mering. Hampp.

Krieg, S. (2015). Spitzenkarrieren im deutschen Krankenhaus. Masterarbeit. Universität Heidelberg.

Kühn, H. (2008). Ökonomisierung im Krankenhaus. In M. Gerhardt, S. Kolb, I. Bode, T. Kaiser, C. Wolf, \& K. Klein (Hrsg.), Medizin und Gewissen: Im Streit zwischen Markt und Solidarität. Dokumentation des Internationalen IPPNW-Kongresses, 20.-22. Oktober 2006 in Nürnberg (S. 285328). Frankfurt a.M.: Mabuse.

Marrs, K. (2007). Ökonomisierung gelungen, Pflegekräfte wohlauf? WSI Mitteilungen, 60, 502-507.

Marrs, K. (2008). Arbeit unter Marktdruck. Die Logik der ökonomischen Steuerung in der Dienstleistungsarbeit. Berlin: edition sigma.

Martinz, T. (2015). Ärztestreiks: Rechtliche Konsequenzen drohen. In Kurier.at. 15.01.2015. Abgerufen von http://kurier.at/chronik/oesterreich/aerztestreik-rechtliche-konsequenzen-drohen/108.349.938 
Mayntz, R., \& Rosewitz, B. (1988). Ausdifferenzierung und Strukturwandel des deutschen Gesundheitssystems. In: R. Mayntz, B. Rosewitz, U. Schimank, \& R. Stichweh (Hrsg.), Differenzierung und Verselbständigung. Zur Entwicklung gesellschaftlicher Teilsysteme (S. 117-172). Frankfurt a.M.: Campus.

Meyer, B. (2013). FMH-Positionspapier zu Bonusvereinbarungen in Spitälern unterstützt Ärztinnen und Ärzte bei Vertragsverhandlungen. Abgerufen von http://www.fmh.ch/files/pdf14/Boni_-_die_Position_der_FMH_SAEZ_2013_51-522.pdf

Müller, N. (2008). Krankenhausträger im internationalen Vergleich Deutschland, Österreich und Schweiz. Eine Bestandsaufnahme unter besonderer Berücksichtigung von Non Profit Organisationen. Diplomarbeit. Hochschule für Wirtschaft und Umwelt, Nürtingen.

Neumann, S. (2005). Non Profit Organisationen unter Druck. Eine Analyse des Anpassungsverhaltens von Organisationen des Gesundheitswesens und der Sozialen Dienste in der Freien Wohlfahrtspflege. München, Mering: Hampp.

Papouschek, U. (2011). Umstrukturierungen im Krankenhaus und ihre Auswirkungen auf die Arbeitsbedingungen. FORBA-Forschungsbericht 5/2011. Wien.

Pichelbaur E. (2014). Protest der Ärzte: Krieg mit dem Skalpell. ZEIT ONLINE, 30.01.2014. Abgerufen von http://www.zeit.de/2014/06/oesterreich-aerzte-streik

Pohlmann, M., Bär, S., \& Valarini, E. (2014). The analysis of collective mindsets: Introducing a New Method of Institutional Analysis in Comparative Research. Revista de Sociologica e Politica, 22 (52), 7-25. doi:10.1590/1678-987314225202

Pöttler, G. (2014). Gesundheitswesen in Österreich. Organisation, Leistungen, Finanzierung und Reformen übersichtlich dargestellt (2. überarbeitete Aufl.). Wien: Goldegg Verlag.

Raible, C., \& Leidl, R. (2004). Bietet die Kooperationsforschung Ansätze zur Erklärung des Wandels auf dem deutschen Krankenhausmarkt. Das Gesundheitswesen, 66, 707-715. doi:10.1055/s-2004-813777

Rothgang, H. (2006). Die Regulierung von Gesundheitssystemen in vergleichender Perspektive: Auf dem Weg zur Konvergenz? In C. Wendt, \& C. Wolf (Hrsg.), Soziologie der Gesundheit. Sonderheft der Kölner Zeitschrift für Soziologie und Sozialpsychologie (S. 298-319). Wiesbaden: VS Verlag für Sozialwissenschaften.

Salfeld, R., Hehner, S., \& Wichels, R. (2009). Modernes Krankenhausmanagement. Konzepte und Lösungen (2. aktualisierte und erweiterte Aufl.). Berlin, Heidelberg: Springer. doi:10.1007/978-3-642-36132-6

Schmid, A., Cacace, M., Götze, R., \& Rothgang, H. (2010). Explaining Health Care System Change: Problem Pressure and the Emergence of "Hybrid" Health Care Systems. Journal of Health Politics, Policy and Law, 35, 455-486. doi:10.1215/03616878-2010-013

Schmitz, C., \& Berchtold, P. (2016). Zur Anatomie medizinischer Prozesse im zeitgenössischen Krankenhauswesen. In I. Bode \& W. Vogd (Hrsg.), Mutationen des Krankenhauses. Soziologische Diagnosen in organisations- und gesellschaftstheoretischer Perspektive (S. 87-102). Wiesbaden: Springer VS. doi:10.1007/978-3-658-11853-2_5

Schott, H. (2006). Aktuelles Gelöbnis der Ärzte. Deutsches Ärzteblatt, 103, 2178.

Schultheis, F., \& Gemperle, M. (2014). Das Gesundheitswesen im Spannungsfeld von Gemeinwohlorientierung und betriebswirtschaftlichen Imperativen. In J. Flecker, F. Schultheis, \& B. Vogel (Hrsg.), Im Dienste öffentlicher Güter. Metamorphosen der Arbeit aus der Sicht der Beschäftigten (S. 23-29). Berlin: edition sigma. doi:10.5771/9783845268989-23

Schweizerische Sanitätsdirektorenkonferenz (2002). Empfehlungen zur zukünftigen Ausgestaltung der Spitalarzt-Abgeltungen. Abgerufen von http://www.gdk-cds.ch/fileadmin/pdf/aktuelles/empfehlungen/chfaz17.10.02-d.pdf

Scott, R. W. (2001). Institutions and Organizations (2. Aufl.). Thousand Oaks: Sage. 
Scott, R. W., Ruef, M., Mendel, P. J.,\& Caronna, C.A. (2000). Institutional Change and Healthcare Organizations. From Professional Dominance to Managed Care. Chicago: University of Chicago Press. doi: $10.2307 / 3094816$

Siegrist, J. (2012). Die ärztliche Rolle im Wandel. Bundesgesundheitsblatt - Gesundheitsforschung Gesundheitsschutz, 55, 1100-1105. doi:10.1007/s00103-012-1527-y

Simon, M. (2001). Die Ökonomisierung des Krankenhauses. Der wachsende Einfluss ökonomischer Ziele auf patientenbezogene Entscheidungen. Veröffentlichungsreihe der Arbeitsgruppe Public Health Wissenschaftszentrum Berlin für Sozialforschung. Berlin.

Simon, M. (2013a). Das Gesundheitssystem in Deutschland. Eine Einführung in Struktur und Funktionsweise (4. überarbeitete und erweiterte Aufl.). Bern: Huber.

Simon, M. (2013b). Das deutsche DRG-System. Grundsätzliche Konstruktionsfehler. Deutsches Ärzteblatt, 110, A1782-6

Simon, M. (2016). Die ökonomischen und strukturellen Veränderungen des Krankenhausbereichs seit den 1970er Jahren. In I. Bode, \& W. Vogd (Hrsg.), Mutationen des Krankenhauses. Soziologische Diagnosen in organisations- und gesellschaftstheoretischer Perspektive (S. 29-45). Wiesbaden: Springer VS. doi:10.1007/978-3-658-11853-2_2

Statistisches Bundesamt (2016). Fachserie 12, Reihe 6.1.1 Gesundheit. Grunddaten der Krankenhäuser. Wiesbaden.

Trinczek, R. (2010). Betriebliche Regulierung von Arbeitsbeziehungen. In F. Böhle, G. G. Voß, \& G. Wachtler (Hrsg.), Handbuch Arbeitssoziologie (S. 841-872). Wiesbaden: VS Verlag für Sozialwissenschaften. doi:10.1007/978-3-531-92247-8_28

Vera, A. (2009). Die „Industrialisierung” des Krankenhauswesens durch DRG-Fallpauschalen - eine interdisziplinäre Analyse. Das Gesundheitswesen, 71, e10-e17. doi:10.1055/s-0028-1102941

Vogd, W. (2011). Zur Soziologie der organisierten Krankenbehandlung. Weilerswist: Velbrück Wissenschaft.

Wilkesmann, M. (2016). Von Fürsten zu Knechten? Aktuelle Transformationsprozesse in der Organisation Krankenhaus am Beispiel der Ärzteschaft. In I. Bode, \& W. Vogd (Hrsg.), Mutationen des Krankenhauses. Soziologische Diagnosen in organisations- und gesellschaftstheoretischer Perspektive (S. 207-228). Wiesbaden: Springer VS. doi:10.1007/978-3-658-11853-2_11 


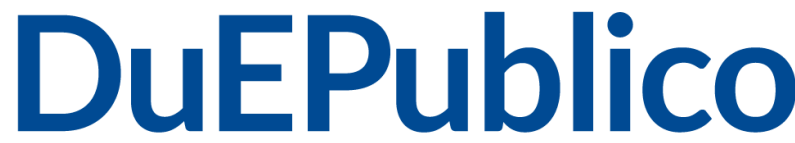

Duisburg-Essen Publications online
UNIVERSITĀT

DESISEN R G

offen im Denken

$\mathbf{U b} \mid \begin{aligned} & \text { universitäts } \\ & \text { bibliothek }\end{aligned}$

Dieser Text wird über DuEPublico, dem Dokumenten- und Publikationsserver der Universität Duisburg-Essen, zur Verfügung gestellt. Die hier veröffentlichte Version der EPublikation kann von einer eventuell ebenfalls veröffentlichten Verlagsversion abweichen.

DOI: $\quad 10.3224$ /indbez.v24i4.03

URN: urn:nbn:de:hbz:464-20200702-162717-9

Dieses Werk kann unter einer Creative Commons Namensnennung

- Weitergabe unter gleichen Bedingungen 4.0 Lizenz (CC BY-SA

4.0) genutzt werden. 